\title{
Effect of 3-Dimensional Airfoil Shape on Unsteady Fluid Forces in Pitching Motion*
}

\author{
Hiroaki HASEGAWA**, Jun WATANABE*** and Kazuo MATSUUCHI**** \\ ${ }^{* *}$ Graduate School of Engineering and Resource Science, Akita University \\ 1-1 Tegata gakuen-machi, Akita-shi, Akita, Japan \\ E-mail: hhasegaw@mech.akita-u.ac.jp \\ ${ }^{* * *}$ Quality Assurance Center, DENSO CORPORATION \\ 1-1 Showa-cho, Kariya, Aichi, Japan \\ ${ }^{* * *}$ Graduate School of Systems and Information Engineering, University of Tsukuba \\ 1-1-1 Tennoudai,Tsukuba, Ibaraki, Japan
}

\begin{abstract}
A quasi-steady-state approach towards predicting the fluid forces acting on a hand under unsteady conditions yielded errors. The actual motion of a hand in swimming is obviously unsteady, and time-dependent fluid forces must be considered. The purpose of this study is to investigate the relationship between unsteady fluid forces and the vortex behaviors of a three-dimensional airfoil during pitch-oscillating motion. A flow visualization technique was used to examine the flow field near the airfoil edge in a wind tunnel test. The unsteady fluid forces were affected by the shedding behavior of vortices from the airfoil edge during the pitching oscillation, and the flow structure due to the vortex behavior was strongly affected by the reduced frequency.
\end{abstract}

Key words: Vortex, Unsteady Flow, Fluid Force, Pitching Oscillation, Wake, Swimming

\section{Introduction}

Studies of unsteady propulsion system of birds, insects, and fish are few and inconclusive. The wings of travelling birds and insects execute complex motions whose most obvious component is flapping, whereas for a fishtail the most obvious component is pitching. In recent years, considerable research efforts in a number of institutions have been devoted to advancing understanding of the propulsive mechanism of flapping wings. It has been noted that the unsteady fluid force plays an important role in biological flight. A quasi-steady approach used to predict the fluid force in flapping flight yielded errors in predicting the fluid forces acting on a wing, suggesting that flight is impossible. However, because biological flight does occur, the effect of unsteady fluid forces must be important to the flight mechanism. These forces must also be considered when estimating the propulsive force of swimmers doing the front crawl. The propulsive force obtained through hand movements is larger than that generated by foot movements. That is, the propulsive force generated by hand motion is dominant in the front crawl. The question of whether propulsion in swimming is primarily due to lift or drag appeared to have been settled in the early 1970s. Before then, it was believed that the best way to propel the body forward was to pull the hand directly backward to use drag forces. The first important contribution related to the mechanism of propulsion in a swimming stroke was made by Counsilman ${ }^{(1)}$. That mechanism has been examined by dividing the force into two components: a lift component normal to the hand motion and a drag one parallel to it. He pointed out the

Received 25 Mar. 2010 (No. T1-08-0375) Eng., Vol.74, No.747, B (2008), pp.2327-2335 (Received 28 Apr., 2008) [DOI: $10.1299 /$ jst.5.270]

Copyright $\odot 2010$ by JSME 
importance of the lift force relative to that of the drag force. The actual motion of a hand in swimming is obviously unsteady, and the time-dependent fluid forces, called dynamic lift, have to be considered. In addition, when predicting the hand force in swimming, the quasi-steady-state approach, which depends on the assumption that the flow at each instant is nearly steady, has led to errors in predicting the fluid forces acting on a hand under unsteady conditions. Quasi-steady analysis underestimates the fluid forces ${ }^{(2)}$.

In a previous study, flow field measurements around the hands of subjects swimming in a flume and wind tunnel tests using an airfoil model simulating a hand were carried out to elucidate unsteadiness in propulsion in swimming ${ }^{(3),(4)}$. In general, there are many parameters to be considered for the unsteady phenomena, and therefore it is difficult to elucidate unsteady mechanisms. Complex parameters affecting unsteady phenomena can easily be changed in a wind tunnel test. To investigate the unsteady fluid forces on a human hand, the three-dimensional characteristics of the test model must be considered. In the present study, the relationship between unsteady fluid forces and vortex behaviors is investigated using a discoid airfoil simulating a swimmer's hand. The unsteady fluid forces and vortical flow field were measured during sinusoidal pitching motion, which represents the fundamental unsteady motion. The experiments were carried out for pitching motion around a stall angle of attack under stationary conditions at which a significant improvement in the maximum lift coefficient has been observed ${ }^{(4)}$. Furthermore, the experiments using a rectangular airfoil with the NACA0012 profile were also performed to investigate the effect of the three-dimensional airfoil model's shape on unsteady fluid forces.

\section{Experimental Apparatus and Method}

\subsection{Experimental apparatus}

Figure 1 shows a schematic of the experimental setup and the coordinate system used to describe the flow field. The test section inlet dimensions were $500 \times 500 \mathrm{~mm}$, and the

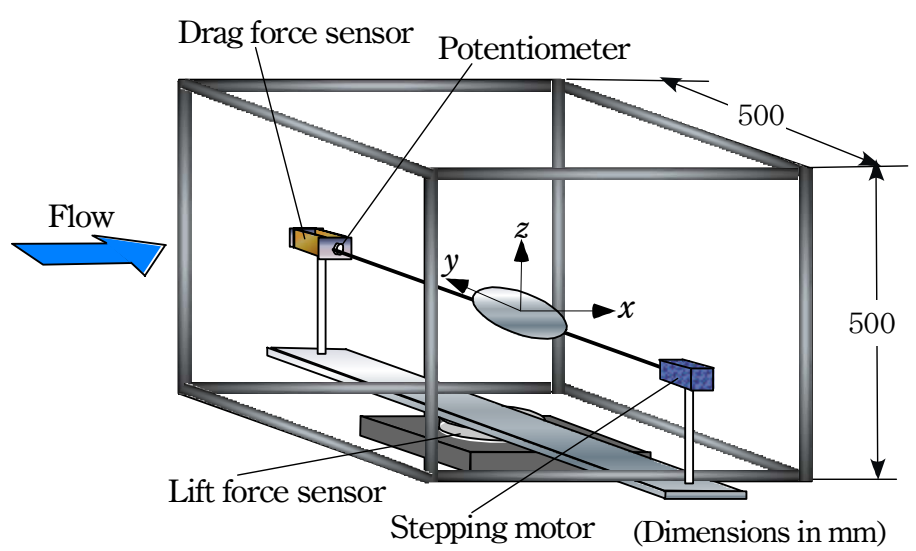

Fig.1 Schematic of experimental set-up.

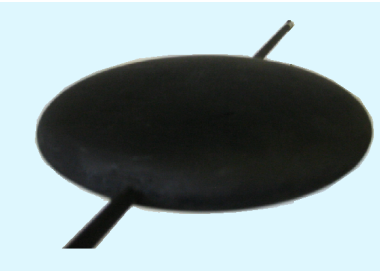

(a) Discoid airfoil

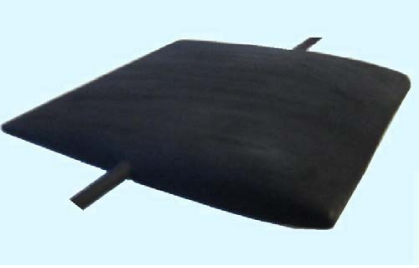

(b) NACA0012

Fig. 2 Test model. 
freestream turbulence intensity was less than 0.2 percent within the operating range. The origins of coordinates $x, y$, and $z$ are defined as the center of the model. The test models, a discoid airfoil and a rectangular airfoil, have NACA0015 and NACA0012 profiles, respectively. Schematics of these models are shown in Fig. 2. The size of the discoid airfoil is estimated by simulating a human hand. The discoid and rectangular airfoils each have a chord $c$ of $150 \mathrm{~mm}$ and a span of $150 \mathrm{~mm}$, and their maximum thicknesses were $37.5 \mathrm{~mm}$ and $22.5 \mathrm{~mm}$, respectively. The airfoil edge was of a smoothed, half-round shape. Pitching motion with a sinusoidal wave was achieved for both models using a five-phase stepping motor with $0.072 \mathrm{deg}$ per 1 step around its mid-chord axis. The measurement error of a model's angle of attack can be evaluated within an error of $\pm 0.5 \%$ using a potential meter. The fluid forces acting on the model were measured using a ring structure balance system. The balance could simultaneously detect all of the lift and drag as functions of time during pitch-oscillating motion. The balance system was described in a previous report ${ }^{(4)}$, and hence its details are omitted here.

\subsection{Experimental method}

The freestream Reynolds number was defined as $R e=c U_{0} / v$, where $U_{0}$ is the freestream velocity, and $v$ is the kinematic viscosity of air. The experiments described here were performed for $R e=7.5 \times 10^{4}$, which corresponds to the Reynolds number range for a swimmer's hand. The fluid force measurements can be evaluated within an error of $0.5 \%$ and 3\% under stationary and unsteady conditions, respectively. The pitching motion is defined by a sinusoidal wave function, and the angle of attack $\alpha$ of the model varied with the function $\alpha=A \sin (2 \pi f t)+\alpha_{c}$, where $A$ is the amplitude, $f$ is the oscillation frequency, and $\alpha_{c}$ is the angle of the pitching center.

The flow field was measured using a particle image velocimetry (PIV) method and hot-wire anemometry. The latter can provide high resolution when the shape and strength of the vortices in the wake are measured for several angles of attack of the pitching model. Velocity measurements in the $y-z$ plane were performed at equal intervals of $10 \mathrm{~mm}$ in both $y$ and $z$ directions using an X-type hot wire probe supported by a three-axis traverse unit. The hot-wire anemometry was synchronized with the pitching motion. The flow field measurements around the test model were carried out by a PIV method, because the hot wire probe cannot take measurements near the surface of the pitching model. The PIV system used here is identical to that introduced by Matsuuchi ${ }^{(3)}$, and therefore the details are omitted here. The flow field is estimated using the ensemble average velocity during several pitching cycles.

\section{Results and Discussion}

\subsection{Fluid force characteristics under stationary conditions}

Figure 3 shows the lift and drag curves under stationary conditions. The static forces are measured at regular intervals of $1.0 \mathrm{deg}$. Upstroke and downstroke indicated in the figure denote the increment and decrement of the angle of attack, respectively. For the discoid airfoil, the maximum lift coefficient is 0.67 and the static stall angle is $25 \mathrm{deg}$. The lift coefficient decreases abruptly after a stall. The lift curve is similar to that of a leading-edge stall, which is caused by abrupt separation of the laminar boundary layer near the leading edge without subsequent reattachment, abruptly decreasing the lift value. The angle of attack in the stall and stall recovery differs between upstroke and downstroke. A clockwise hysteresis loop exists in the lift curve, and a counter-clockwise loop exists in the drag curve. In a leading-edge stall, the laminar boundary layer separation occurs before the stall, and the separated flow reattaches within a short distance as a turbulent boundary layer. The region underlying the separated flow, which exists between the points of separation and 


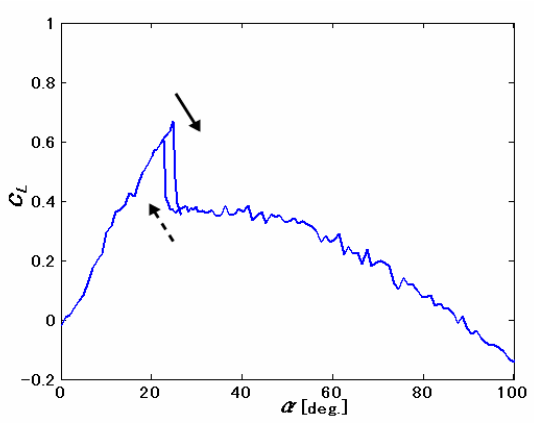

(a) $\mathrm{C}_{\mathrm{L}}$ curve for discoid airfoil

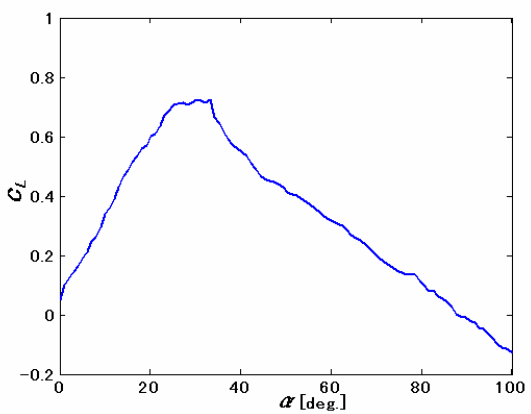

(c) $\mathrm{C}_{\mathrm{L}}$ curve for NACA0012

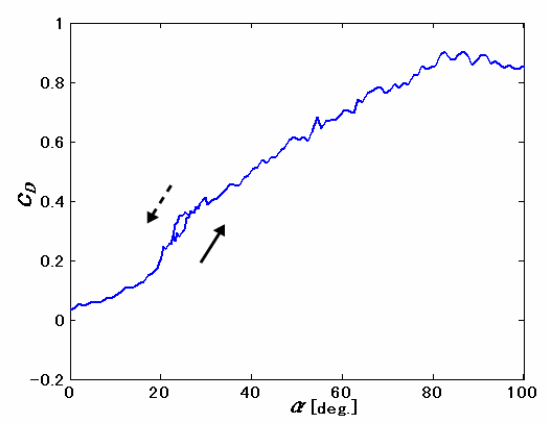

(b) $\mathrm{C}_{\mathrm{D}}$ curve for discoid airfoil

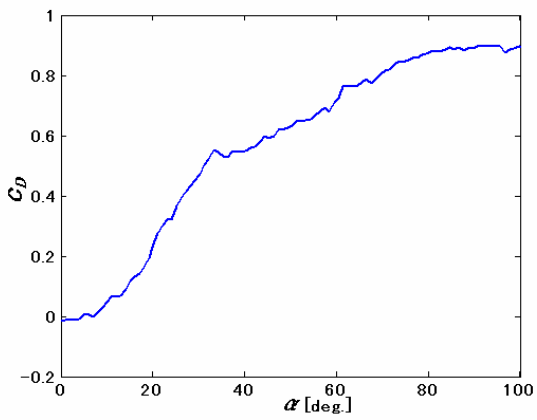

(d) $C_{D}$ curve for NACA001

Fig. 3 Lift and drag curves under stationary conditions. Solid and dotted arrows denote upstroke and downstroke, respectively.

reattachment and is set into circulating motion, is commonly referred to as a short bubble or bubble. With an increase in angle of attack, the bubble contracts in length until it suddenly breaks down or bursts, causing flow separation without subsequent reattachment. This causes an abrupt loss of lift at the stall. The region underlying the separated flow is then referred to as a long bubble. The long bubble grows rapidly with increasing angle of attack until it extends over the entire airfoil chord. To recover from a stall, flow reattachment must occur. However, the angle of attack needed for recovery from the stall is delayed because recovery from this stall state depends on the previous flow conditions. Namely, in the stall state downstroke, the lift coefficient is lower and the drag coefficient is larger than that in upstroke. Therefore, clockwise hysteresis appears in the lift curve, and counter-clockwise hysteresis appears in the drag curve.

For the model with the NACA0012 profile, the maximum lift coefficient is 0.72 , and the static stall angle is $32 \mathrm{deg}$. The abrupt decrement in lift coefficient is not observed just after a stall, in contrast to the case of the discoid airfoil model. Furthermore, no hysteresis appears in the lift and drag curves. This lift curve is similar to that of a thin-airfoil stall preceded by laminar separation near the leading edge with turbulent reattachment at a point that moves progressively rearward with an increase in the angle of attack.

The lift coefficient characteristics of the two models have the same tendency: the lift value increases linearly as the angle of attack increases up to the stall, and the lift curve slopes in the two models have almost the same inclination. This result agrees well with the wing theory showing that the lift curve is not affected by airfoil profile for a two-dimensional airfoil. However, the stall angle for a three-dimensional airfoil is larger than that for a two-dimensional airfoil. At a Reynolds number of $1.8 \times 10^{5}$, the stall angle of attack is $16 \mathrm{deg}^{(5)}$ and $10 \mathrm{deg}^{(6)}$ for two-dimensional airfoils with the NACA0015 and NACA0012 profiles, respectively. The Reynolds number disagrees sharply with the case in this experiment, but it can be concluded that the stall angle of attack for a three-dimensional airfoil becomes larger than that for a two-dimensional airfoil in the two airfoil profiles because the downwash flow is induced by the longitudinal vortex pair shed from the airfoil edge of the three-dimensional airfoil. The effective angle of attack is decreased by this 
downwash flow, and therefore the stall angle of attack for the three-dimensional airfoil increases relative to that of a two-dimensional airfoil with the same profile.

\subsection{Flow field under stationary conditions}

Figures 4 and 5 show contour maps of the vorticity and velocity vectors in the wake of the discoid and NACA0012 airfoils, respectively. The color bar in each figure indicates the strength of the vorticity. The vortical field in the wake was measured using an X-type hot-wire probe supported by a three-axis computer-controlled traverse unit. Streamwise vorticity $\omega$ is given by

$$
\omega=\frac{\partial w}{\partial y}-\frac{\partial v}{\partial z}
$$

Here, the vorticity is defined as positive for vortices of counter-clockwise rotation (viewed from downstream). Positive vortices were depicted on the right side of all the contour maps. The vorticity is calculated by the velocity in the $y-z$ plane measured at equal intervals of 10 $\mathrm{mm}$ in both the $y$ and $z$ directions. The dotted lines in these figures denote the airfoil profile viewed from downstream for better understanding of the airfoil position at the measured angle of attack. A counter-rotating vortex pair is observed downstream of the airfoil before the stall. On the other hand, beyond the stall angle of attack, positive and negative vortices become weak and are elongated along the airfoil edge. For the discoid airfoil, the strength and shape of the longitudinal vortices at the same angle of attack differ significantly between upstroke and downstroke. This difference in the flow field corresponds to the existence of hysteresis in the lift curve. At an angle of attack of 25.2 deg during upstroke,

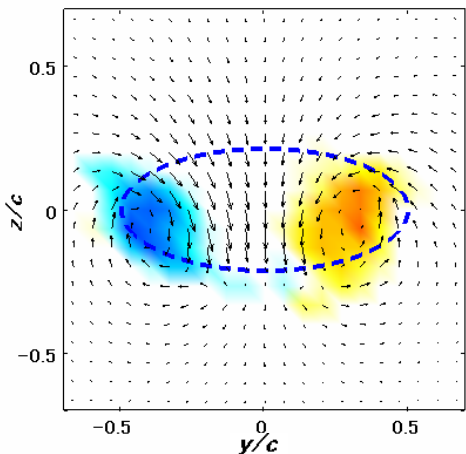

(a) $\alpha=25.2 \mathrm{deg}$ (upstroke)

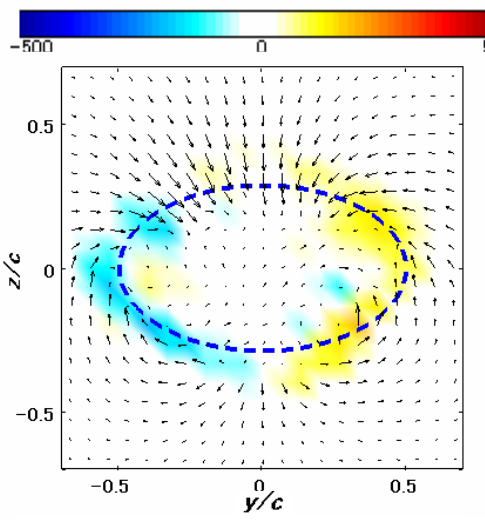

(b) $\alpha=35.2 \mathrm{deg}$

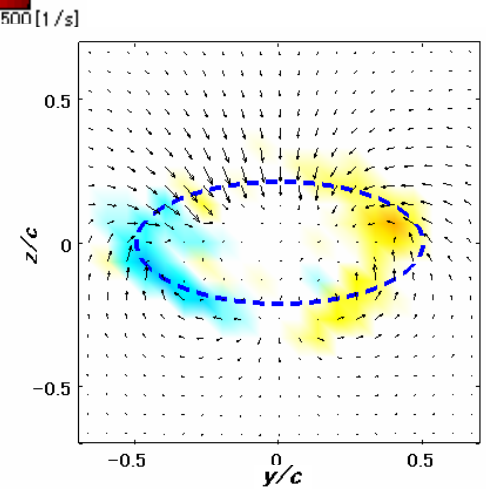

(c) $\alpha=25.2 \mathrm{deg}$ (downstroke)

Fig. 4 Density map of vorticity and secondary flow vectors under stationary conditions for discoid airfoil.

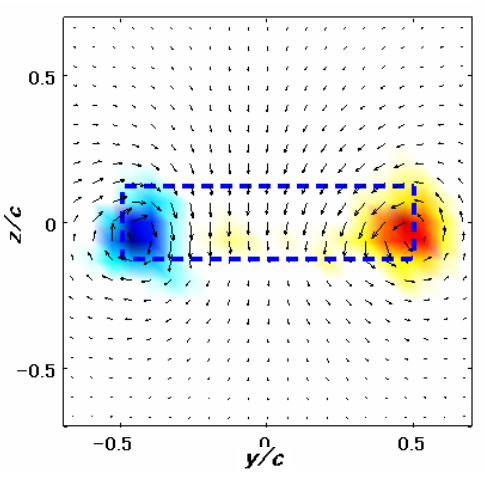

(a) $\alpha=14.5 \mathrm{deg}$

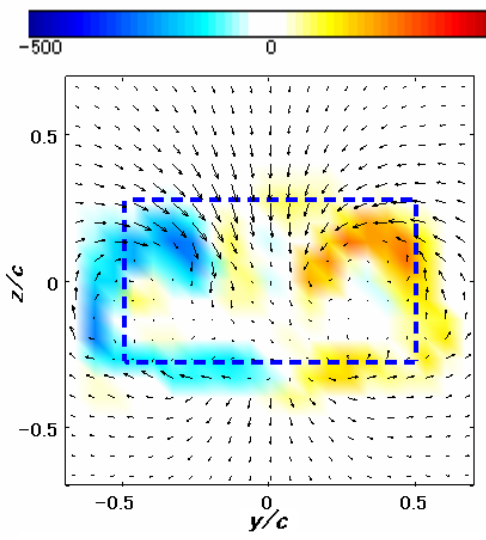

(b) $\alpha=32.8 \mathrm{deg}$ (upstroke)

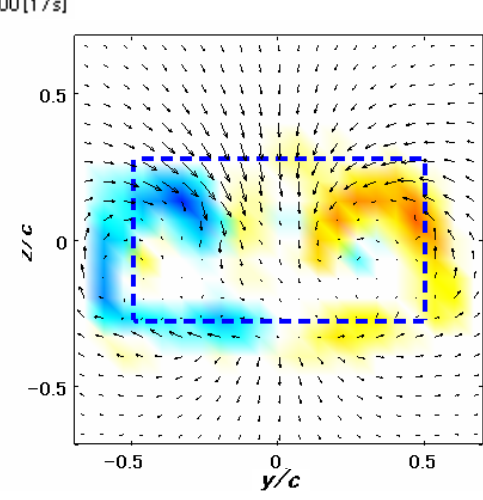

(c) $\alpha=32.8 \mathrm{deg}$ (downstroke)

Fig. 5 Density map of vorticity and secondary flow vectors under stationary conditions for NACA0012. 
the lift coefficient is large because the airfoil is not stalling, and the flow vectors indicate a strong downwash. The generation of lift force around the model is confirmed by the presence of this strong downward flow. In contrast, at an angle of attack of 25.2 deg during downstroke, stall recovery is not attained [see Fig. 3(a)]. The longitudinal vortices in the wake are weakened and vertically elongated. Moreover, no downward flow was observed in the wake.

For the NACA0012 airfoil, the shape and strength of vortices at an angle of attack of $32.8 \mathrm{deg}$ were similar during upstroke and downstroke. No hysteresis in the lift curve is observed for this airfoil [see Fig. 3(c)], and the flow field shows no obvious difference between upstroke and downstroke. At an angle of attack of $32.8 \mathrm{deg}$, at which the lift coefficient decreases, the longitudinal vortices in the wake of the airfoil elongate along the airfoil edge. However, the vorticity decrement after a stall is smaller than in the case of the discoid airfoil. Vortex breakdown is promoted because of the abrupt loss of lift at the stall for the discoid airfoil. In contrast, the vortex does not rapidly break down for NACA0012 airfoil, and a region of strong vorticity exists inside the vortex that elongates along the airfoil edge. The lift curve showed no sudden drop after the stall, unlike the case of the discoid airfoil. For a three-dimensional airfoil, it was confirmed that the fluid forces acting on an airfoil were affected by the behavior of the pair of longitudinal vortices in the wake.

\subsection{Unsteady fluid force characteristics}

Figures 6 and 7 show the unsteady lift and drag curves during pitching motion. The pitching amplitude $A$ was fixed at $A=10 \mathrm{deg}$ in this experiment. The angles of the pitching center $\alpha_{c}$ are $25.2 \mathrm{deg}$ and $32.0 \mathrm{deg}$ for the discoid and NACA0012 airfoils, respectively. These angles correspond to the static stall angle of attack for each model. Pitching frequencies of $f=0.6,1.0,2.0,4.0$, and $6.0 \mathrm{~Hz}$ are used in this experiment. In these figures, solid and dotted arrows indicate upstroke (increment of angle of attack) and downstroke (decrement of angle of attack) during the pitching motion.

For the discoid airfoil, the maximum lift coefficient for several pitching frequencies under unsteady conditions is greater than that under stationary conditions. For $f=0.6 \mathrm{~Hz}$, a stall occurs in upstroke. Subsequently, even if the angle of attack is lower than the static stall angle, lift recovery is not observed. The unsteady lift curve shows significant hysteresis between upstroke and downstroke. On the other hand, at $f=6.0 \mathrm{~Hz}$, an abrupt loss of lift at the stall is not observed in upstroke. The maximum lift coefficient and the stall angle increase with increasing pitching frequency. For $f=6.0 \mathrm{~Hz}$, the lift curve shows slight hysteresis between upstroke and downstroke, and the loss of lift against the steady lift coefficient during downstroke, which appears in the $f=0.6 \mathrm{~Hz}$ case, is not confirmed. For $f$ $=6.0 \mathrm{~Hz}$, no stall is observed during downstroke.

During upstroke, the effective angle of attack of the airfoil becomes smaller than the geometric angle of attack because the induced flow, which is due to the pitching motion of the airfoil, inclines downward against the freestream direction. That is, downwash is created, and the local airflow is deflected downward in the vicinity of the airfoil by the angle of the pitching airfoil during upstroke. This is the induced angle of attack. The airfoil section itself is then responding to an effective angle of attack equal to the geometric angle of attack minus the induced angle of attack. Moreover, this downward velocity keeps the vortex from detaching from the upper surface of the airfoil, and the distance between the leading edge vortex and the upper surface is shortened while the separated vortex is convected downstream because the geometric angle of attack of the airfoil increases during upstroke. The leading edge vortex is easily attached to the upper surface of the airfoil by the pitching motion. This airfoil movement promotes an increase in the circulation around the airfoil. Therefore, the lift increases beyond the static stall angle for a pitching airfoil. 


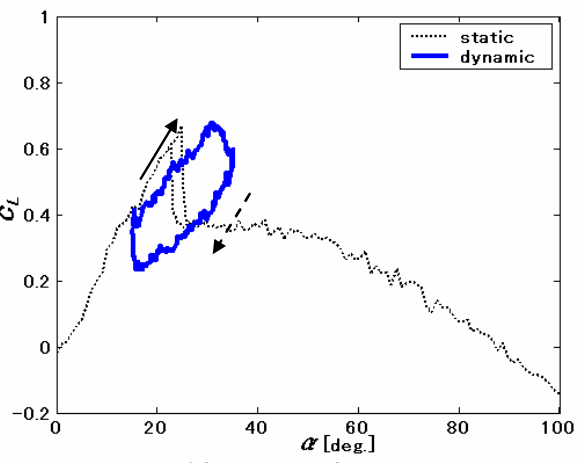

(a) Lift curve $(f=0.6 \mathrm{~Hz})$

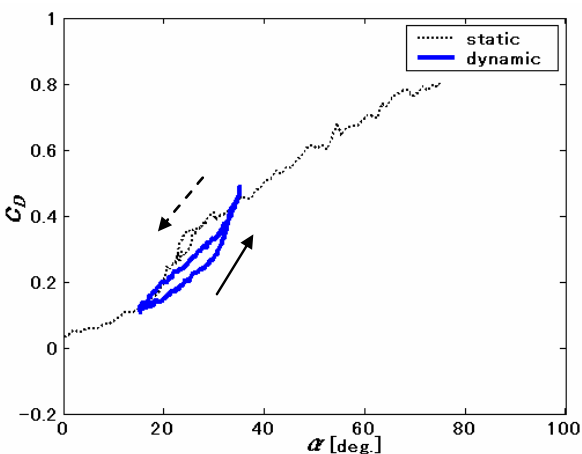

(c) Drag curve $(f=0.6 \mathrm{~Hz})$

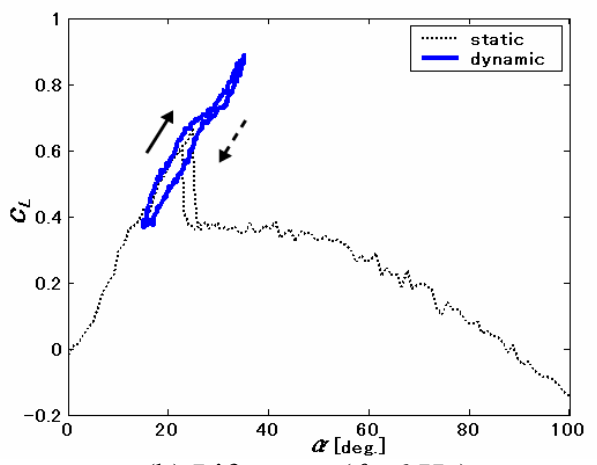

(b) Lift curve $(f=6 \mathrm{~Hz})$

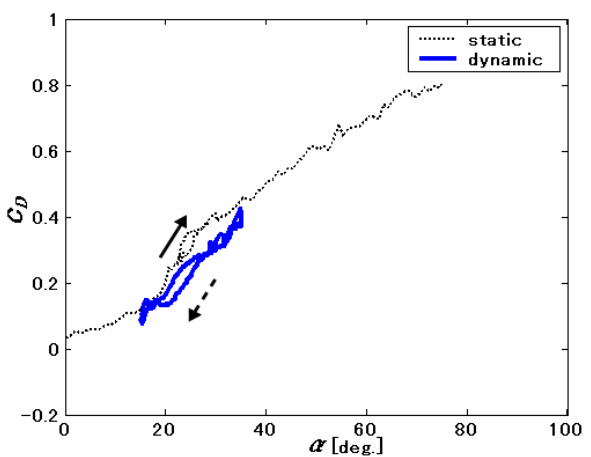

(d) Drag curve $(f=6 \mathrm{~Hz})$

Fig. 6 Hysteresis loop of dynamic lift and drag for discoid airfoil $\left(\alpha_{c}=25.2^{\circ}\right)$.

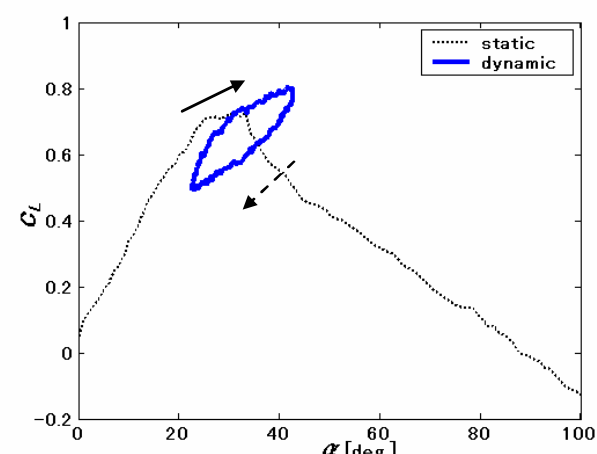

(a) Lift curve $(f=0.6 \mathrm{~Hz})$

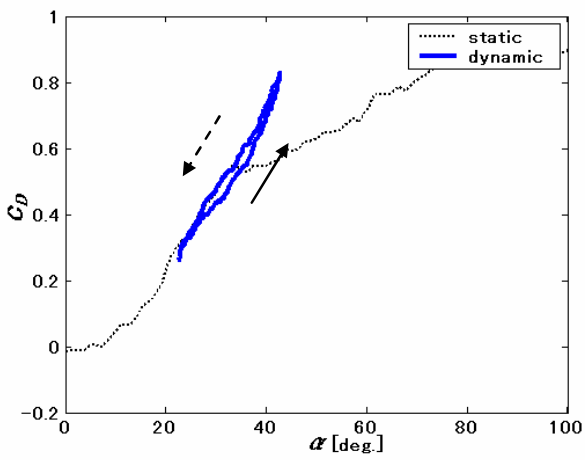

(c) Drag curve $(f=0.6 \mathrm{~Hz})$

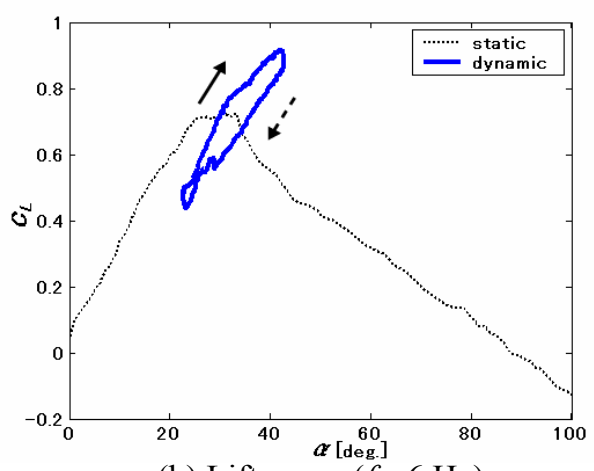

(b) Lift curve $(f=6 \mathrm{~Hz})$

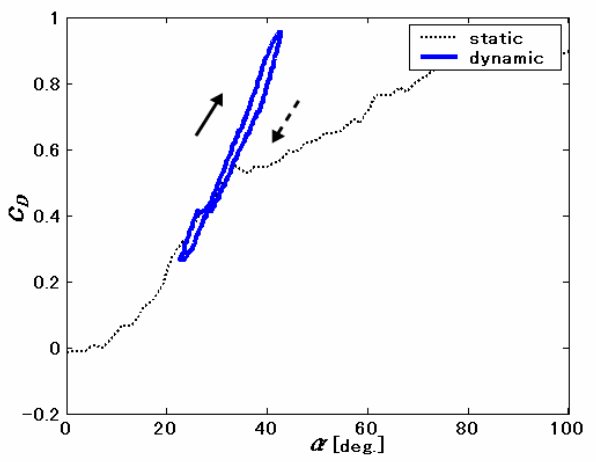

(d) Drag curve $(f=6 \mathrm{~Hz})$

Fig. 7 Hysteresis loop of dynamic lift and drag for NACA0012 $\left(\alpha_{c}=32.0^{\circ}\right)$.

In contrast, during downstroke, the effective angle of attack of the airfoil becomes larger than the geometric angle of attack because the induced flow, which is due to the pitching motion, inclines upward against the freestream direction. Furthermore, this airfoil 
movement promotes a decrease in the circulation around the airfoil. The flow field change around the airfoil is obvious at a high pitching frequency, and no stall is observed during upstroke for $f=6 \mathrm{~Hz}$. After that, during downstroke, the lift curve returns along the path it followed during upstroke and the hysteresis between upstroke and downstroke is small as a result of the flow around the airfoil, with no stall condition or increment of the effective angle of attack.

In the drag curve during the pitching motion, hysteresis between upstroke and downstroke is observed, and it becomes smaller with increasing pitching frequency. This behavior is similar to that of the lift curve. For $f=6 \mathrm{~Hz}$, the drag value under unsteady conditions is less than that under stationary conditions. A considerable rise in the drag value is seen at the stall under stationary conditions. At the angle of the pitching center defined as the static stall angle of attack, the slope of the drag curve changes. On the other hand, the rise in induced drag with the increment of the angle of attack is suppressed by the stall delay, and hysteresis appears, with a slope along the slope of the steady drag curve before the stall. Therefore, the drag coefficient during the pitching motion does not significantly increase, in contrast to the stationary cases. For the discoid airfoil, the lift coefficient increases, but the drag coefficient barely increases.

Figure 7 confirms that the maximum lift coefficient and stall angle delay are observed in the dynamic lift curves for the NACA0012 airfoil. The lift curve slope increases gradually before the stall owing to the characteristics of thin-airfoil stall. That is, the lift curve changes in slope at the angle of attack at which the (separation) bubble above the airfoil surface is generated, and stalling occurs after the slope decreases slightly. The slope of the dynamic lift curve during upstroke is similar to that before the static lift curve slope decreases prior to the static stall. This result suggests that the pitching motion can maintain the flow above the airfoil surface in the state preceding separation. The dynamic lift curve slope during upstroke changes at an angle of attack up to the static stall angle, which corresponds to the pitching center's angle of attack.

In the drag curves under stationary conditions, the drag coefficient increases as a result of the stall and then becomes gradual. On the other hand, the dynamic drag increases abruptly because of the stall, and the abrupt increment of the slope of the drag curve is maintained after the stall. The drag coefficient under unsteady conditions is significantly larger than that under stationary conditions, in contrast to the discoid airfoil case. For the NACA0012 airfoil, the longitudinal vortices do not suddenly break down after the stall; therefore, a remarkable increase in the dynamic drag coefficient occurs because the flow depends strongly on the induced drag. In addition, the hysteresis in the dynamic drag curves is small, in contrast to the case of the discoid airfoil. Not only the stall delay and maximum lift coefficient, but also the drag coefficient increase for the NACA0012 airfoil.

The ratio of the lift-to-drag ratio under unsteady conditions to that under stationary conditions for several reduced frequencies is shown in Fig. 8. The lift-to-drag ratio is calculated using the maximum values of lift and drag in one pitching cycle. The results for different angles of attack of the pitching center are also plotted in Fig. 8. The ratio increases with increasing reduced frequency for the discoid airfoil. For the NACA0012 airfoil, the ratio decreases slightly with increasing reduced frequency, and is always less than 1 when the pitching center is set to the static stall angle of attack. That is, the lift-to-drag ratio under pitching motion is less than that in the stationary state. The ratio of the dynamic and static lifts becomes larger for the discoid airfoil than for the NACA0012 airfoil. Furthermore, the ratio of the dynamic and static drags decreases and increases for the discoid airfoil and the NACA0012 airfoil, respectively. However, at $\alpha_{\mathrm{c}}=24.5 \mathrm{deg}$, the ratio increases with increasing reduced frequency for the NACA0012 airfoil, because the drag decreases with increasing reduced frequency due to the change in the pitching center angle.

In summary, unsteadiness in the pitching motion of the discoid airfoil, which has the 


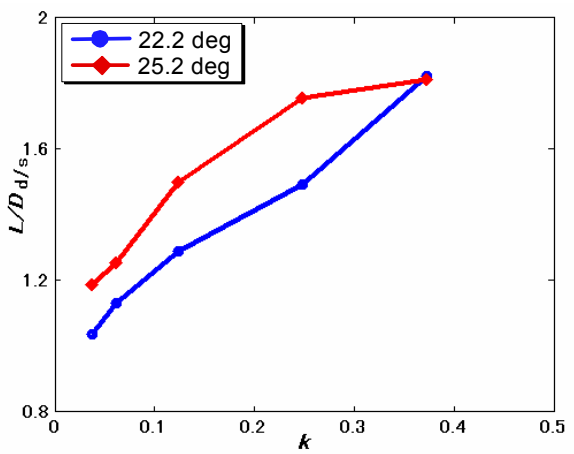

(a) Discoid airfoil

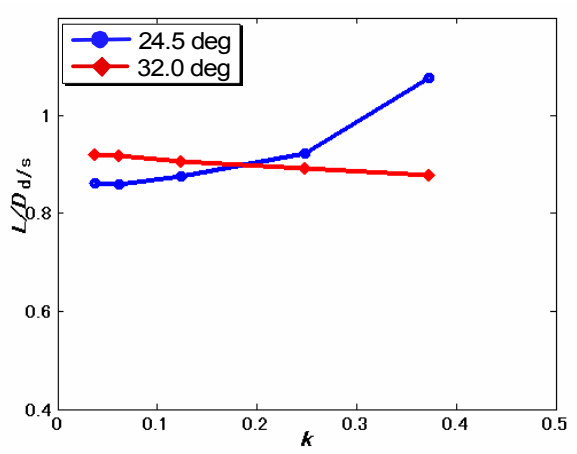

(b) NACA0012

Fig. 8 Lift-to-drag ratio between dynamic and stationary conditions $\left(k=\pi f c / U_{0}\right)$.

characteristics of leading-edge stall, effectively promotes stall delay and high lift performance. For the NACA0012 airfoil, which shows no abrupt loss of lift at the stall, the effect of unsteadiness is attained by properly selecting the pitching center angle. In other words, the effect of unsteadiness during the pitching motion is affected by the airfoil's stalling characteristics under stationary conditions and the pitching center angle.

\subsection{Flow fields around the airfoil during pitching motion}

For the discoid airfoil, which shows an improved maximum lift coefficient and lift-to-drag ratio under pitching motion, the vortical fields in the wake of the airfoil are shown in Figs. 9 and 10. These fields were measured by an X-type hot-wire probe. The figures also show the secondary flow vectors. The dotted lines indicate the airfoil profile at the measured angle of attack viewed from downstream. The non-dimensional time $t^{\prime}$ is defined as the time divided by one cycle of the pitching motion. For $f=1 \mathrm{~Hz}$, the vortical field differs between upstroke and downstroke. At $t^{\prime}=0.4$ during downstroke, the longitudinal vortices elongate along the airfoil edge, and a strong vortex pair does not appear. In contrast, at $t^{\prime}=0.6$ during upstroke, which is the same angle of attack as at $t^{\prime}=$ 0.4 , a strong vortex pair can be observed. This vortex pair grows along the airfoil edge as the angle of attack increases, and reaches a maximum strength at $t^{\prime}=0.9$, when the maximum lift coefficient is obtained. Hysteresis between upstroke and downstroke appears for the discoid airfoil, as mentioned earlier, and therefore the vortical fields in the wake of the airfoil differ in tendency between upstroke and downstroke.

On the other hand, vortex breakdown is not observed for $f=6 \mathrm{~Hz}$. At $t^{\prime}=0.1$, beyond the angle of attack at the instant of changing from upstroke to downstroke, a vortex pair appears in the wake. For $f=1 \mathrm{~Hz}$, at $t^{\prime}=0.1$ the vortex elongates along the airfoil edge, and the vortices weaken. For $f=6 \mathrm{~Hz}$, the vortex pair retains its strength with decreasing angle of attack because it is produced during the downstroke just after the instant of changing from upstroke to downstroke. Therefore, at $t^{\prime}=0.4$, just before the flow change occurs from downstroke to upstroke, the vortex pair exists, unlike the case for $f=1 \mathrm{~Hz}$, in which the vortex breaks down.

The flow fields above the airfoil surface, measured by a PIV method, are shown in Fig. 11. For $f=1 \mathrm{~Hz}$, the flow separated from the leading edge passes downstream without subsequent reattachment at $t^{\prime}=0.1$. This non-reattached flow is commonly associated with the flow field for which the vortex elongates along the airfoil profile, and the vortex pair breaks down in the $y-z$ plane, as mentioned above. Subsequently, at $t^{\prime}=0.9$ with the same angle of attack as at $t^{\prime}=0.1$, flow is observed above the airfoil's upper surface. At this angle of attack, a strong vortex pair appears in the wake of the airfoil. When the vortex resulting from leading edge separation is attached to the upper side of the airfoil surface, the vortex shed from the airfoil edge gives rise to a vortex pair. That is, the behavior of the 
vortex pair in the wake is strongly affected by the flow above the upper surface of the airfoil.

The flow above the upper surface of the airfoil exists in the downstream direction with no separation from the airfoil surface for $f=6 \mathrm{~Hz}$; the flow over a portion of the airfoil surface does not lead to a complete separation of flow at the instant of changing from upstroke to downstroke. If the effective angle of attack increases during downstroke, the lift force can be maintained because the separated flow does not appear before downstroke. The time during which the effective angle of attack increases is shorter at a higher pitching frequency. The lift force begins to increase during upstroke as a result of reattachment of the flow above the upper surface.

\section{Conclusions}

In this study, the unsteady fluid forces acting on a discoid airfoil simulating a human hand are measured, and the differences in the effect of unsteadiness on the discoid airfoil and the NACA0012 airfoil, which has different steady stall characteristics, are investigated.

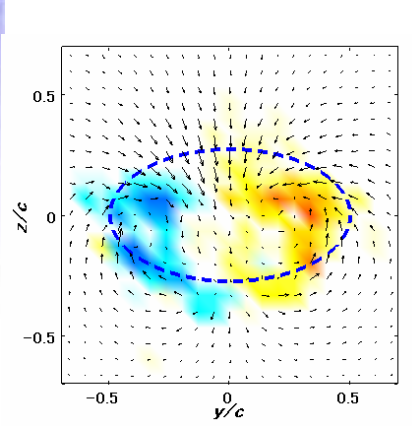

(a) $t^{\prime}=0.1$

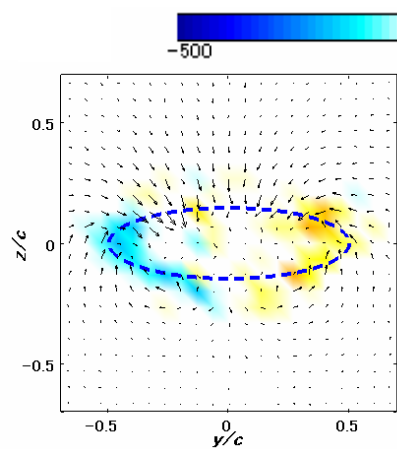

(b) $t \stackrel{\prime \prime}{=} 0.4$

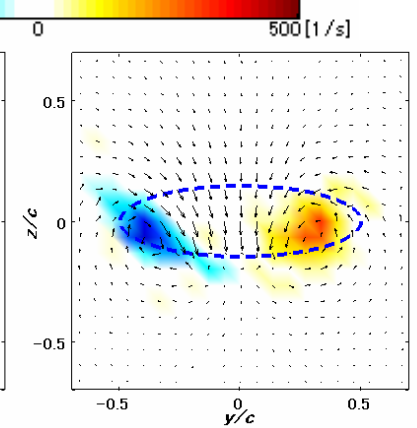

(c) $t^{\prime \prime}=0.6$

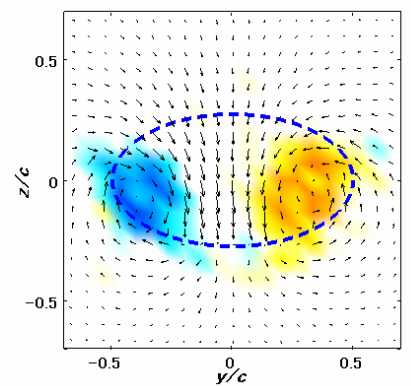

(d) $t^{\prime}=0.9$

Fig. 9 Density map of vorticity and secondary flow vectors at $x / c=0.5$ for discoid airfoil $\left(f=1.0 \mathrm{~Hz}, \alpha_{c}=25.2^{\circ}\right)$.

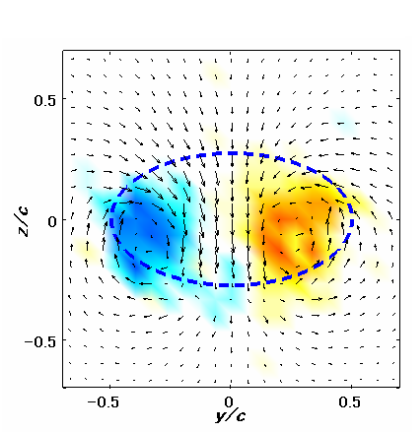

(a) $t^{\prime}=0.1$

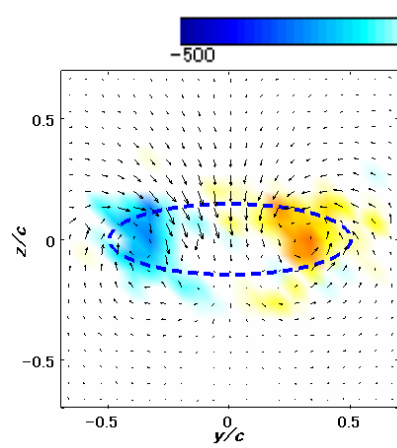

(b) $t^{\prime}=0.4$

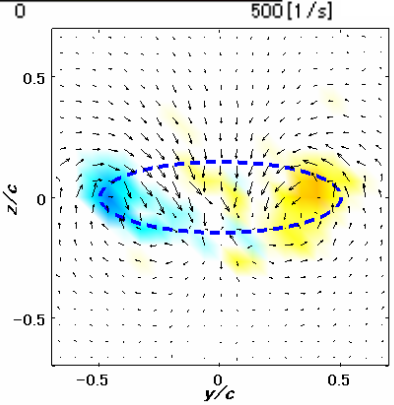

(c) $t^{\prime}=0.6$

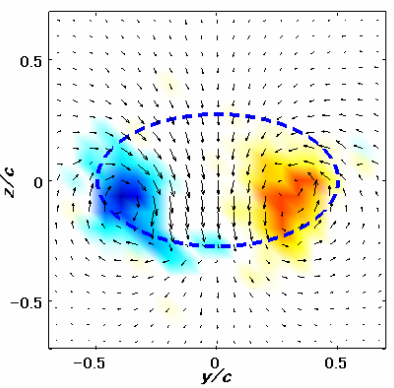

(d) $t^{\prime}=0.9$

Fig. 10 Density map of vorticity and secondary flow vectors at $x / c=0.5$ for discoid airfoil $\left(f=6.0 \mathrm{~Hz}, \alpha_{c}=25.2^{\circ}\right)$.

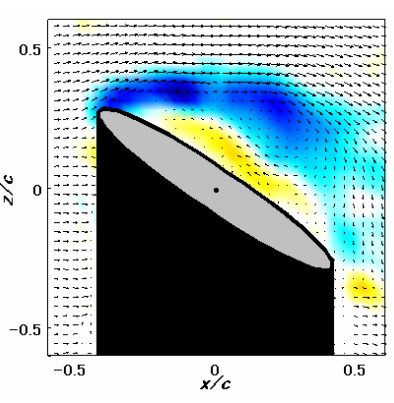

(a) $t^{\prime}=0.1$

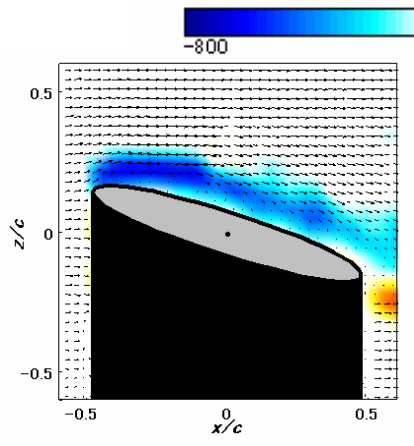

(b) $t \stackrel{\prime \prime}{=} 0.4$

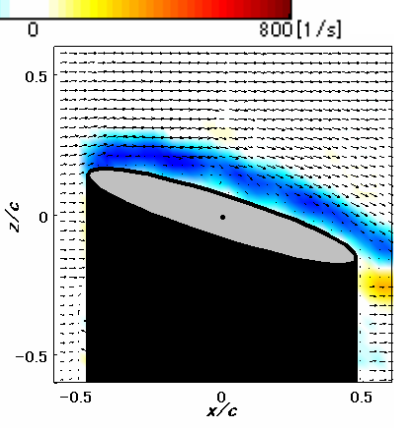

(c) $t^{\prime}=0.6$

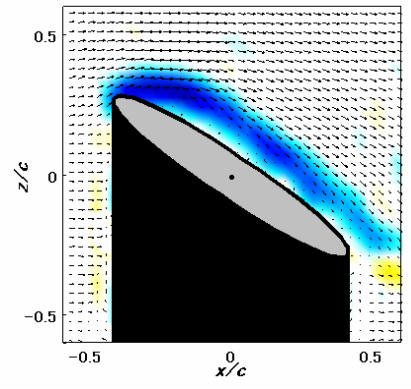

(d) $t^{\prime}=0.9$

Fig. 11 Flow fields around discoid airfoil at $y / \mathrm{c}=0\left(f=1.0 \mathrm{~Hz}, \alpha_{c}=25.2^{\circ}\right)$. 
Furthermore, the relationship between the dynamic lift and vortex behavior is also considered. The results are summarized as follows:

(1) For the discoid airfoil, the fluid force characteristics under stationary conditions show an abrupt loss of lift at the stall; a clockwise hysteresis loop is observed in the lift curve, and a counterclockwise hysteresis loop is observed in the drag curve. For the NACA0012 airfoil, the lift gradually decreases as the angle of attack increases beyond the stall angle of attack, and no hysteresis loop is observed in the lift and drag curves.

(2) The effect of unsteadiness during the pitching motion is affected by the static stall characteristics. For the discoid airfoil, the hysteresis in the lift curve decreases with increasing pitching frequency, and the ratio of the lift-to-drag ratios under the unsteady and stationary conditions increases. Furthermore, this phenomenon is only slightly affected by the pitching center's angle of attack.

(3) For the NACA0012 airfoil, the ratio of the lift-to-drag ratios under unsteady and stationary conditions is not affected by the pitching frequency during the pitching motion near the static stall angle of attack. The ratio is less than 1, and unsteadiness has no observable effect. However, the ratio of the lift-to-drag ratios can be increased with increasing pitching frequency by properly changing the pitching center's angle of attack.

(4) A vortex pair exists in the wake when the vortex separated from the leading edge moves downstream with no detachment on the upper surface of the airfoil. The vortex pair in the wake becomes elongated along the airfoil edge when the vortex separated from the leading edge causes flow separation without subsequent reattachment, and the vortex breaks down at the stall.

\section{Acknowledgments}

This study was supported by a Grant-in-Aid for Scientific Research ((B)(2) 15300216 and (C)17500411) from the Japan Society for the Promotion of Science.

\section{References}

(1) Counsilman, J. E., The Application of Bernoulli's Principle to Human Propulsion in Water, First International Symposium on Biomechanics of Swimming, Water Polo and Diving, (1970), pp. 59-71.

(2) Berger, M. A. M., Hollander A. P. and De Groot, G., Determining Propulsive Force in Front Crawl Swimming: A Comparison of Two Methods, J. Sport Sciences, 17 (1999), pp. 97-105.

(3) Matsuuchi, K., Yamada, K., Nomura, T., Sakakibara, J., Shintani, H. and Miwa, T., Motion Analysis of Front Crawl Swimmer's Hand and Visualization of Flow Fields Using PIV, Transactions of the Japan Society of Mechanical Engineers, Series B, Vol. 73, No. 734 (2007), pp. 2027-2032.

(4) Hasegawa, H., Odaka, Y. and Matsuuchi, K., Relationship between Dynamic Lift and Streamwise Vortices Acting on a Disk Simulating a Hand of Swimmer, Journal of Japan Society of Fluid Mechanics, Vol. 25, No. 4 (2006), pp. 357-366.

(5) Jumper, E. J., Schreck, S. J. and Dimmick, R. L., Lift-Curve Characteristics for an Airfoil Pitching at Constant Rate, J. Aircraft, 24-10, (1987), pp. 680-687.

(6) Abbott, I. H. and Von Doenhoff, A. E., Theory of Wing Sections, Dover, (1958). 